ARTIGOS / ARTICLES

DOI: $10.5433 / 1679-0367.2021 v 42 n 2 p 187$

\title{
Diagnósticos de enfermagem em recuperação pós-anestésica: intervenções e resultados segundo linguagens padronizadas
}

\author{
Nursing diagnoses in post-anesthetic recovery: outcomes \\ and interventions according to standardized languages
}

\author{
Marina Prearo ${ }^{1}$, Marcelli Cristine Vocci ${ }^{2}$, \\ Cassiana Mendes Bertoncello Fontes ${ }^{3}$
}

\begin{abstract}
Resumo
Introdução: os sistemas de classificação com linguagens padronizadas se estabelecem em um conjunto de conhecimentos estruturados, conceitos fundados de forma lógica e coerente, com base em suas similaridades. Nesse sentido, identificar um perfil junto a populações pode cooperar para uma melhor definição e compreensão situacional para aquela unidade e/ou pacientes. Objetivos: realizar mapeamento cruzado entre os diagnósticos de enfermagem da NANDA-I com os registros manuais de enfermagem em sala de recuperação pós-anestésica; e propor intervenções e resultados, segundo linguagens padronizadas. Método: estudo exploratório, retrospectivo com análise estatística descritiva de registros de enfermagem de 187 pacientes que estiveram hospitalizados no período de junho a julho de 2018, em sala de recuperação pós-anestésica de um hospital oncológico. O mapeamento cruzado foi realizado em três etapas: identificação dos indicadores dos diagnósticos; proposição de intervenções e atividades; e indicadores de resultados. Os dados foram analisados e descritos em frequências absoluta e relativa. Resultados: dos 13 domínios da NANDA-I, cinco foram representados; identificaram-se cinco diagnósticos de risco e 11 com foco no problema; observou-se $100 \%$ de frequência para os diagnósticos de: Risco de aspiração; Risco de infecção; Risco de queda; Capacidade de transferência prejudicada; Mobilidade no leito prejudicada; Integridade da pele/tissular prejudicada e Conforto prejudicado. Conclusões: para os 16 diagnósticos de enfermagem mapeados, foram selecionadas 22 intervenções e 58 atividades; 23 resultados e 48 indicadores de resultados.
\end{abstract}

Palavras-chave: Enfermagem em pós-anestésico; Registro de enfermagem; NANDA-I; Terminologia NIC; Terminologia NOC.

\footnotetext{
${ }^{1}$ Graduação em Enfermagem pela Faculdades Integradas de Jaú (FIJ), Jaú, São Paulo, Brasil. Diretora de Serviços de Saúde da Prefeitura Municipal de Bariri, Bariri, São Paulo, Brasil.

${ }^{2}$ Doutoranda no Programa de Pós-graduação em Enfermagem da Faculdade de Medicina de Botucatu da Universidade Estadual Paulista (FMB-Unesp), Botucatu, São Paulo, Brasil. E-mail: marcellivocci@hotmail.com

${ }^{3}$ Doutorado em Saúde do Adulto pela Escola de Enfermagem da Universidade de São Paulo (EE-USP), São Paulo, São Paulo, Brasil. Professora Assistente Doutora no Departamento de Enfermagem da Faculdade de Medicina de Botucatu da Universidade Estadual Paulista, Botucatu, São Paulo, Brasil.
} 


\begin{abstract}
Introduction: the classification systems with standardized languages are established in a set of structured knowledge, concepts founded in a logical and coherent way, based on their similarities. In this sense, identifying a profile with the populations can cooperate for a better definition and situational understanding for that unit and/or patients. Objectives: to perform cross-mapping between the nursing diagnoses of NANDA-I with the manual nursing records in the post-anesthetic recovery room; and, to propose interventions and outcomes, according to standardized language. Method: exploratory, descriptive and retrospective analysis of the nursing records of 187 patients hospitalized from June to July 2018, in the post-anesthetic recovery room of an oncology hospital. Cross-mapping was carried out in three stages: identification of diagnostic indicators; proposition of interventions and activities; and outcomes indicators. The data were analyzed and described in absolute and relative frequencies. Results: of the 13 NANDA-I domains, five were highlighted; were identified five risk diagnoses and 11 focused on the problem; $100 \%$ frequency was observed for the diagnoses of: Aspiration, infection and falling risk; Impaired transfer capacity; Impaired bed mobility; Impaired skin/tissue integrity and impaired comfort. Conclusions: from the 16 nursing diagnoses mapped, were selected 22 interventions and 58 activities; 23 results and 48 outcomes indicators.
\end{abstract}

Keywords: Postanesthesia nursing; Nursing records; NANDA-I; NIC terminology; NOC terminology.

\section{Introdução}

A Sala de Recuperação Pós-anestésica (SRPA) é uma unidade destinada a pacientes em pós-operatório imediato (POI), período compreendido entre as primeiras vinte e quatro horas após uma intervenção anestésica cirúrgica. A equipe de enfermagem da SRPA tem a finalidade de admitir o paciente, proporcionar cuidado seguro, prevenir e detectar complicações pós-anestésicas e cirúrgicas, assim como realizar o registro da assistência de enfermagem. ${ }^{(1)}$

O Processo de Enfermagem (PE) configura-se como uma metodologia organizacional embasada em princípios científicos, e de reconhecimento legal de acordo com a Resolução do Conselho Federal de Enfermagem n ${ }^{0} 358 / 2009{ }^{(2)}$ que proporciona a melhoria da qualidade da assistência de enfermagem e contribui para a atuação da equipe de forma a promover a segurança do paciente. Na SRPA, o PE, também denominado como Sistematização da Assistência de Enfermagem (SAE), auxilia para que o enfermeiro identifique as prioridades de cada paciente quanto às suas necessidades, como a evolução da consciência, o retorno de reflexos protetores e a estabilidade dos sinais vitais. ${ }^{(3-5)}$
A proposição deste estudo foi embasada nos conceitos da Teoria de Enfermagem de Wanda Aguiar Horta ${ }^{(6)}$ Necessidades Humanas Básicas ${ }^{(7)}$ e na assistência sistematizada com registro de enfermagem baseado em linguagem padronizada. As terminologias padronizadas são: Classificação de Diagnóstico de Enfermagem da NANDA International (NANDA-I), ${ }^{(8)}$ Classificação das Intervenções de Enfermagem, da Nursing Intervention Classification (NIC) ${ }^{(9)}$ e a Classificação dos Resultados de Enfermagem, da Nursing Intervention Classification (NOC). ${ }^{(10)}$

A Taxonomia II da NANDA-I possui 13 Domínios, 46 Classes e 244 diagnósticos de enfermagem (DE), que estruturam-se a partir dos indicadores: características definidoras; fatores relacionados; fatores de riscos; condições associadas e populações de risco. As classificações da NIC e da NOC possuem, respectivamente, um conjunto de Intervenções de Enfermagem (IE), Atividades de Enfermagem (AE), e Resultados de Enfermagem (RE) com indicadores de resultados (IR), a partir da nomeação dos diagnósticos.

Nesse contexto, NANDA, NOC e NIC desempenham importante papel ao descrever, de modo padronizado, elementos de interesse da prática profissional. Os sistemas de classificação 
com linguagens padronizadas se estabelecem em um conjunto de conhecimentos estruturados, conceitos fundados de forma lógica e coerente, com base em suas similaridades. Nesse sentido, identificar um perfil de DE junto a populações pode cooperar para uma melhor definição e compreensão situacional para aquela unidade e/ou pacientes. ${ }^{(11)}$

Em SRPA, o planejamento da assistência de enfermagem deve ser considerado como um instrumento de segurança e qualidade, onde a promoção do cuidado no POI baseado em linguagem padronizada possibilita ao enfermeiro subsidiar ações de cuidado e de gestão, facilitar o dimensionamento de recurso humano e material, fortalecer a educação permanente, e dar visibilidade e autonomia ao processo de trabalho do enfermeiro. ${ }^{(12-13)}$

Desse modo, os objetivos do presente estudo foram: realizar mapeamento cruzado entre os diagnósticos de enfermagem da NANDA-I com os registros manuais de enfermagem em SRPA; e propor intervenções e resultados, segundo linguagens padronizadas.

\section{Método}

\section{Desenho do estudo}

Trata-se de um estudo exploratório, retrospectivo com análise estatística descritiva dos registros de enfermagem de 187 fichas de pacientes admitidos em SRPA no período de junho a julho de 2018, em um hospital de referência em tratamento oncológico no interior do estado de São Paulo, Brasil.

Nesta instituição, local do estudo, os campos para o preenchimento manual da ficha eram: identificação do paciente; sinais vitais; necessidades humanas afetadas; prescrição médica; e anotação de enfermagem. Os registros traziam dados como monitoração dos sinais vitais; ventilação mecânica; resultados de exames; tipos de procedimento realizados; horários de admissão e alta no centro cirúrgico e na SRPA; e os dados subjetivos referiam-se à evolução, história clínica e intercorrências.

\section{População e amostra}

Para composição da população do estudo foi solicitado um relatório ao setor responsável com as seguintes informações: horários de admissão; alta; tempo total de permanência do paciente na SRPA; e unidade de destino. Foram identificadas 264 fichas de pacientes. Os critérios de inclusão foram: registro de admissão na SRPA entre $6 \mathrm{~h}$ e $12 \mathrm{~h}$, de segunda-feira a sábado, e fichas com completude de preenchimento. Assim, excluíram-se 72 fichas que estavam incompletas e cinco que não foram localizadas, totalizando-se 187 fichas incluídas na amostra do estudo.

\section{Coleta e análise de dados}

As 187 fichas foram analisadas e extraídos os dados de relevância. Dois instrumentos de coleta foram construídos e utilizados: o instrumento A, para a coleta dos dados objetivos e subjetivos; e o instrumento B, para a descrição dos resultados do mapeamento cruzado entre o registro das fichas e os indicadores dos diagnósticos de enfermagem, segundo a NANDA-I. ${ }^{(8)}$

A análise descritiva dos dados constituiu-se de três etapas: a) análise dos dados do instrumento $\mathrm{A}$; b) análise do instrumento $\mathrm{B}$, com os resultados do mapeamento cruzado, ${ }^{(14-15)}$ que originou a listagem dos DE construídos em consenso entre as pesquisadoras; e c) proposição e atribuição de intervenções e de resultados. Os dados foram descritos segundo as frequências absoluta e relativa.

A nomeação do título diagnóstico pelas pesquisadoras foi baseada na análise e identificação de dados objetivos e subjetivos registrados nas fichas, e realizado o mapeamento cruzado com a NANDA-I. Esse processo consistiu em identificar termos ou palavras levantados no instrumento A e que possuíam semelhança com a estrutura da taxonomia. Dessa maneira, foram relacionados e descritos os títulos diagnósticos no instrumento $\mathrm{B}$.

Para a seleção das intervenções e atividades de enfermagem utilizou-se a NIC, e por meio da 
NOC, foram identificados os resultados a serem atingidos a partir do título diagnóstico. A listagem com os DE foi submetida à análise estatística descritiva e demonstrada em frequências absoluta e relativa.

\section{Aspectos éticos}

O estudo foi aprovado sob parecer do comitê de ética em pesquisa (CEP) da Fundação Doutor Amaral Carvalho, número 2.678.773; com dispensa do Termo de Consentimento Livre e Esclarecido (TCLE).

\section{Resultados}

Com a elaboração da listagem dos DE, apresentaram-se cinco diagnósticos de risco e 11 com foco no problema. A Tabela 1 demonstra as frequências absoluta e relativa dos diagnósticos de risco e com foco no problema, de acordo com os domínios da NANDA-I.

Tabela 1 - Frequências absoluta e relativa dos domínios e respectivos diagnósticos de enfermagem de risco e com foco no problema. Botucatu, São Paulo, Brasil, 2019.

\begin{tabular}{|c|c|c|c|c|}
\hline \multicolumn{2}{|c|}{ Domínios } & Diagnóstico de enfermagem & $\mathbf{n}$ & $\%$ \\
\hline Domínio 2 & Nutrição & Risco de glicemia instável & 17 & 9,0 \\
\hline Domínio 4 & Atividade/repouso & Capacidade de transferência prejudicada & 187 & 100,0 \\
\hline Domínio 4 & Atividade/repouso & Mobilidade no leito prejudicada & 187 & 100,0 \\
\hline Domínio 4 & Atividade/repouso & Ventilação espontânea prejudicada & 9 & 5,0 \\
\hline Domínio 5 & Percepção/cognição & Confusão aguda & 3 & 2,0 \\
\hline Domínio 11 & Segurança/proteção & Risco de aspiração & 187 & 100,0 \\
\hline Domínio 11 & Segurança/proteção & Risco de infecção & 187 & 100,0 \\
\hline Domínio 11 & Segurança/proteção & Risco de queda & 187 & 100,0 \\
\hline Domínio 11 & Segurança/proteção & Risco de hipotermia perioperatória & 87 & 47,0 \\
\hline Domínio 11 & Segurança/proteção & Integridade da pele prejudicada & 187 & 100,0 \\
\hline Domínio 11 & Segurança/proteção & Integridade tissular prejudicada & 187 & 100,0 \\
\hline Domínio 11 & Segurança/proteção & Desobstrução ineficaz de vias aéreas & 115 & 61,0 \\
\hline Domínio 11 & Segurança/proteção & Hipotermia & 100 & 53,0 \\
\hline Domínio 12 & Conforto & Conforto prejudicado & 187 & 100,0 \\
\hline Domínio 12 & Conforto & Dor aguda & 62 & 33,0 \\
\hline Domínio 12 & Conforto & Náusea & 7 & 4,0 \\
\hline
\end{tabular}

Fonte: as autoras.

Optou-se por apresentar nos Quadros 1 e 2 respectivas proposições de intervenções, atividaos DE que obtiveram $100 \%$ de frequência e suas des, resultados e indicadores. ${ }^{(9-10)}$ 
Quadro 1 - Diagnósticos de enfermagem de risco e respectivas intervenções, atividades, resultados de enfermagem e indicadores de resultados. Botucatu, São Paulo, Brasil, 2019.

\begin{tabular}{|c|c|c|}
\hline Diagnósticos & & ervenções, atividades, resultados e indicadores \\
\hline \multirow{20}{*}{$\begin{array}{l}\text { Risco de } \\
\text { aspiração }\end{array}$} & Intervenções & Atividades \\
\hline & \multirow{5}{*}{ Controle de vias aéreas } & Encorajar respiração lenta e profunda. \\
\hline & & Verificar a necessidade de realizar aspiração. \\
\hline & & Administrar oxigênio, conforme apropriado. \\
\hline & & Posicionar para aliviar dispneia e prevenir aspiração. \\
\hline & & Monitorar estado respiratório e a oxigenação. \\
\hline & \multirow{3}{*}{ Controle do vômito } & $\begin{array}{l}\text { Identificar fatores que possam causar ou contribuir para episódios } \\
\text { de vômitos. }\end{array}$ \\
\hline & & $\begin{array}{l}\text { Assegurar que medicamentos antieméticos sejam administrados } \\
\text { para prevenção de vômitos, quando possível. }\end{array}$ \\
\hline & & Monitorar quanto à sensação de plenitude, náuseas e vômitos. \\
\hline & $\begin{array}{l}\text { Monitoração } \\
\text { neurológica }\end{array}$ & Monitorar nível de consciência. \\
\hline & $\begin{array}{l}\text { Cuidados com sondas } \\
\text { gastrointestinais }\end{array}$ & Monitorar a quantidade, cor e consistência do débito nasogástrico. \\
\hline & Resultados & Indicadores \\
\hline & \multirow{4}{*}{$\begin{array}{l}\text { Recuperação } \\
\text { pós-procedimento }\end{array}$} & Vias aéreas pérvias. \\
\hline & & SPO2 a $92 \%-94 \%$ em ar ambiente. \\
\hline & & Totalmente acordado. \\
\hline & & Náusea e/ou vômito. \\
\hline & \multirow{4}{*}{ Estado da deglutição } & Mantém posição neutra da cabeça e do tronco. \\
\hline & & Controle das secreções orais. \\
\hline & & Tosse. \\
\hline & & Engasgo. \\
\hline \multirow{11}{*}{$\begin{array}{l}\text { Risco de } \\
\text { infecção }\end{array}$} & Intervenções & Atividades \\
\hline & \multirow{3}{*}{$\begin{array}{l}\text { Cuidados com local } \\
\text { da incisão }\end{array}$} & Inspecionar o local da incisão para detecção de anormalidades. \\
\hline & & Observar as características de qualquer secreção. \\
\hline & & Trocar curativo conforme a quantidade de exsudato e drenagem. \\
\hline & \multirow{3}{*}{ Cuidados com lesões } & $\begin{array}{l}\text { Manter técnica asséptica durante a realização do curativo ao cuidar } \\
\text { da lesão. }\end{array}$ \\
\hline & & $\begin{array}{l}\text { Posicionar paciente de modo a evitar a tensão sobre a lesão, conforme } \\
\text { apropriado. }\end{array}$ \\
\hline & & Documentar localização, tamanho e aspecto da lesão. \\
\hline & Supervisão da pele & $\begin{array}{l}\text { Inspecionar a pele e as mucosas quanto à vermelhidão, calor extremo, } \\
\text { edema ou drenagem. }\end{array}$ \\
\hline & Resultados & Indicadores \\
\hline & \multirow{2}{*}{$\begin{array}{l}\text { Integridade tissular: } \\
\text { pele e mucosas }\end{array}$} & Integridade tecidual. \\
\hline & & Lesão na pele e/ou mucosas. \\
\hline
\end{tabular}




\section{Continuação}

\begin{tabular}{|c|c|c|}
\hline \multirow{13}{*}{$\begin{array}{l}\text { Risco de } \\
\text { queda }\end{array}$} & Intervenções & Atividades \\
\hline & \multirow{2}{*}{$\begin{array}{l}\text { Prevenção } \\
\text { contra quedas }\end{array}$} & Identificar os comportamentos e fatores que afetam o risco de quedas. \\
\hline & & $\begin{array}{l}\text { Usar grades laterais com comprimento e altura apropriados } \\
\text { para impedir a queda da cama. }\end{array}$ \\
\hline & \multirow{6}{*}{ Transferência } & Determinar o nível de consciência e a capacidade de cooperar. \\
\hline & & Planejar o tipo e o método de movimentação. \\
\hline & & Determinar a quantidade e o tipo de assistência necessária. \\
\hline & & $\begin{array}{l}\text { Ajustar o equipamento conforme necessário para a altura que facilite } \\
\text { o trabalho e travar todas as rodas. }\end{array}$ \\
\hline & & Transferir o paciente a partir da cama para a maca, usando um lençol. \\
\hline & & $\begin{array}{l}\text { Avaliar o paciente no final da transferência quanto a alinhamento } \\
\text { adequado do corpo, pele exposta desnecessariamente e grades laterais } \\
\text { levantadas. }\end{array}$ \\
\hline & Resultados & Indicadores \\
\hline & Mobilidade & Desempenho no posicionamento do corpo. \\
\hline & $\begin{array}{l}\text { Desempenho } \\
\text { na transferência }\end{array}$ & Transferência de uma superfície à outra enquanto deitado. \\
\hline & Nível de delírio & Agitação. \\
\hline
\end{tabular}

Fonte: as autoras.

Nota-se que no Quadro 1 foram elencados para os diagnósticos de risco: nove intervenções, 25 atividades, seis resultados e nove indicadores.
No Quadro 2, para os diagnósticos com foco no problema foram: 13 intervenções, 33 atividades, 17 resultados e 39 indicadores.

Quadro 2 - Diagnósticos de enfermagem com foco no problema e suas respectivas intervenções, atividades, resultados e indicadores de resultados. Botucatu, São Paulo, Brasil, 2019.

\begin{tabular}{|c|c|c|}
\hline Diagnósticos & & Intervenções, atividades, resultados e indicadores \\
\hline \multirow{9}{*}{$\begin{array}{l}\text { Capacidade de } \\
\text { transferência } \\
\text { prejudicada }\end{array}$} & Intervenções & Atividades \\
\hline & \multirow{6}{*}{$\begin{array}{l}\text { Assistência } \\
\text { no autocuidado: } \\
\text { transferência }\end{array}$} & Determinar a capacidade atual do paciente de autotransferência. \\
\hline & & Selecionar a técnica de transferência apropriada para o paciente. \\
\hline & & Identificar métodos para prevenir lesões durante a transferência. \\
\hline & & Determinar a quantidade e o tipo de assistência necessária. \\
\hline & & Manter o corpo do paciente corretamente alinhado durante os movimentos. \\
\hline & & $\begin{array}{l}\text { Avaliar o paciente no final da transferência quanto a alinhamento } \\
\text { adequado do corpo, pele exposta desnecessariamente e grades laterais } \\
\text { levantadas. }\end{array}$ \\
\hline & Resultados & Indicadores \\
\hline & $\begin{array}{l}\text { Desempenho } \\
\text { na transferência }\end{array}$ & Transferência de uma superfície à outra enquanto deitado. \\
\hline
\end{tabular}




\section{Continuação}

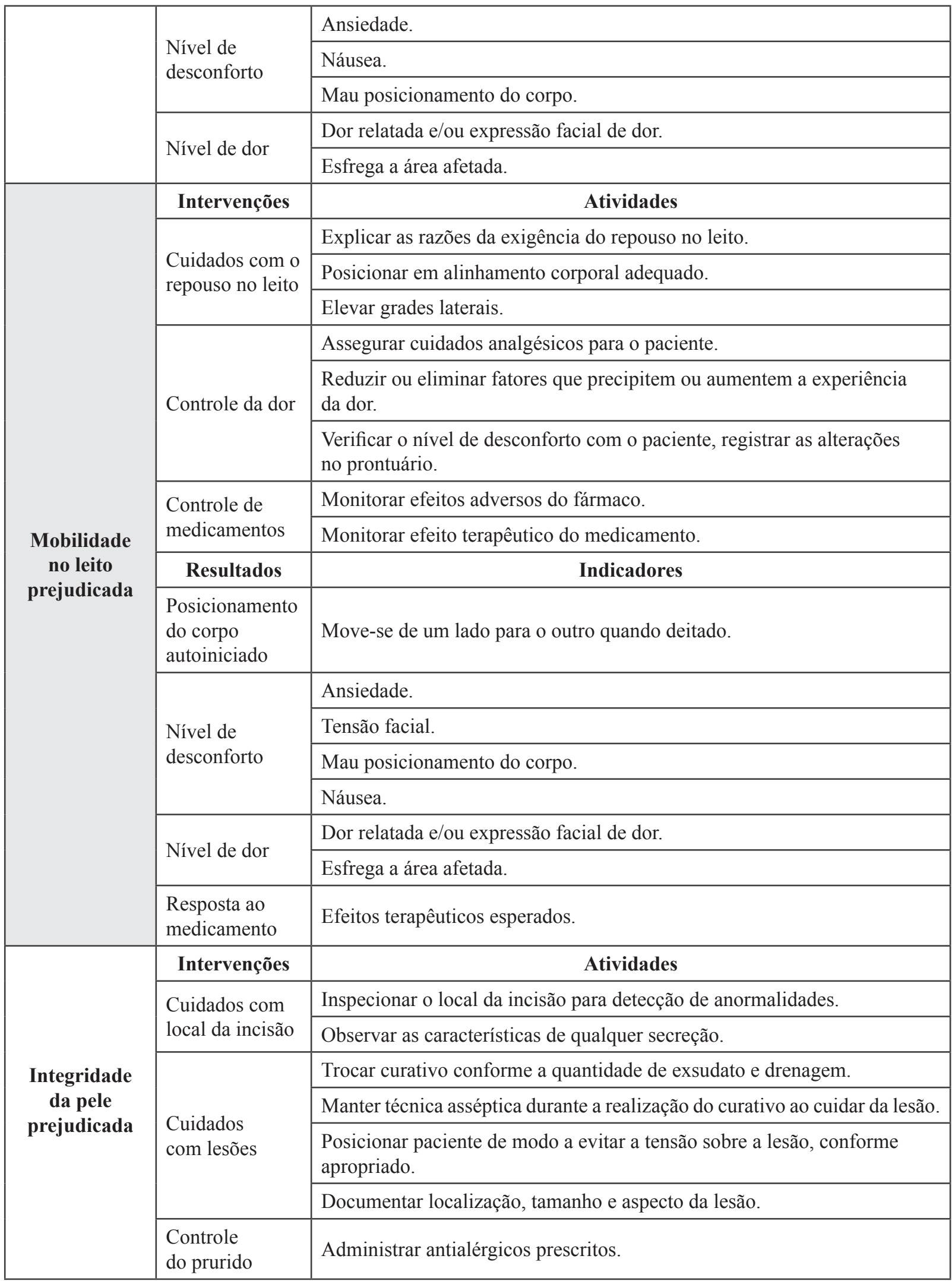




\section{Continuação}

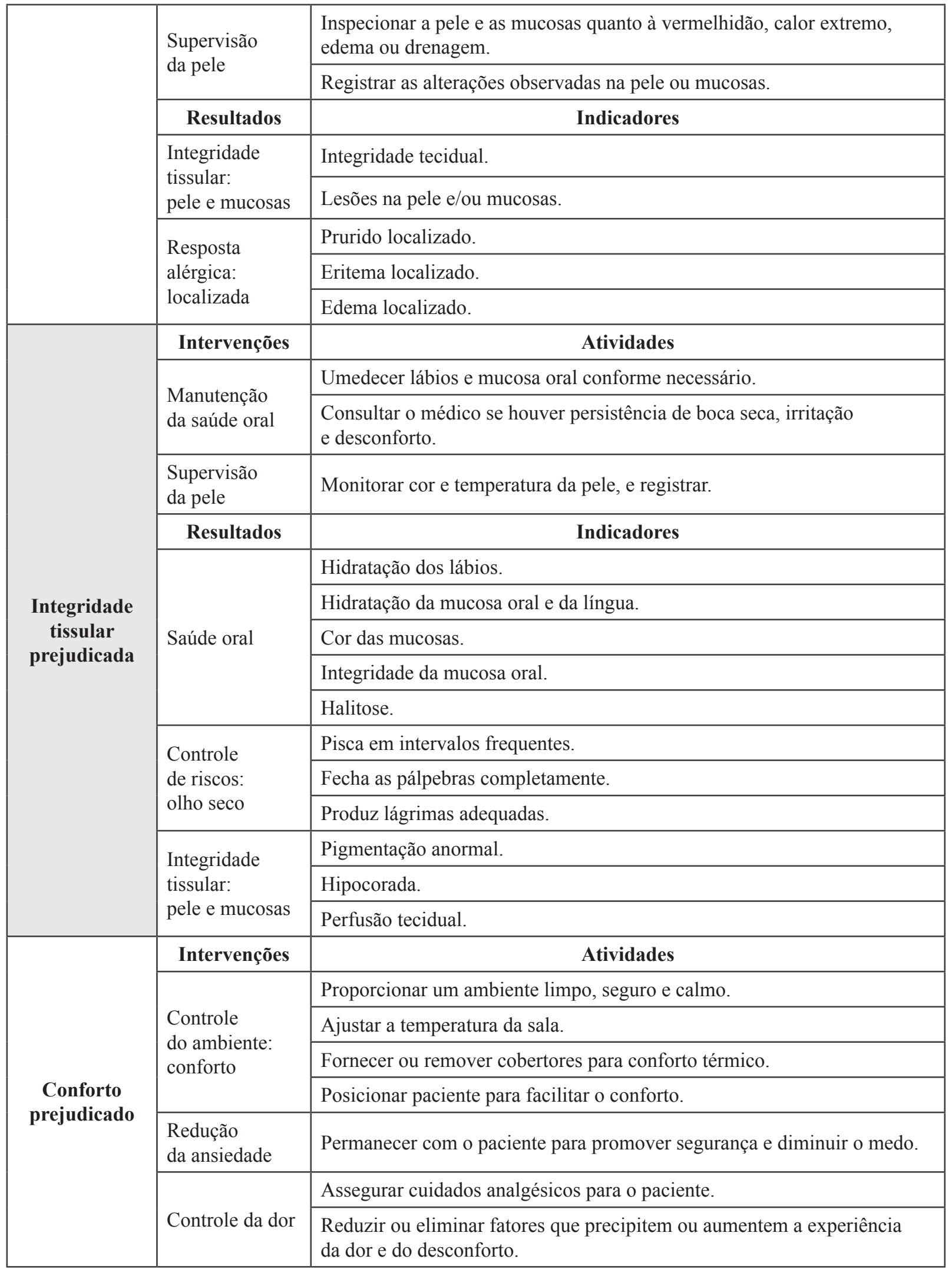




\begin{tabular}{|c|c|}
\hline Resultados & Indicadores \\
\hline \multirow{2}{*}{$\begin{array}{l}\text { Estado } \\
\text { de conforto: } \\
\text { ambiente }\end{array}$} & Temperatura do ambiente. \\
\hline & Cama confortável. \\
\hline \multirow{2}{*}{$\begin{array}{l}\text { Estado } \\
\text { de conforto: } \\
\text { físico }\end{array}$} & Posição confortável. \\
\hline & Dificuldade respiratória. \\
\hline \multirow{2}{*}{$\begin{array}{l}\text { Estado } \\
\text { de conforto: } \\
\text { psicoespiritual }\end{array}$} & Ansiedade. \\
\hline & Medo. \\
\hline \multirow{2}{*}{ Nível de dor } & Dor relatada. \\
\hline & Inquietação. \\
\hline Nível de fadiga & Humor deprimido. \\
\hline
\end{tabular}

Fonte: as autoras.

\section{Discussão}

A não validação por juízes especialistas na temática dos achados do presente estudo é uma limitação, porém os resultados contribuem para a implementação da assistência de enfermagem perioperatória sistematizada, o que pode proporcionar segurança e qualidade no cuidado, minimizar riscos inerentes às condições dessa população, nortear a prática clínica e fortalecer a autonomia profissional do enfermeiro em SRPA.

Os resultados evidenciaram que o Domínio 11 da NANDA-I (Segurança/proteção) obteve maior representatividade de diagnósticos de risco, com foco no problema. Discutem-se a seguir, as relações do perfil de necessidades de cuidados aos pacientes admitidos na SRPA: Risco de aspiração; Risco de infecção; Risco de queda; Capacidade de transferência prejudicada; Mobilidade no leito prejudicada; Integridade da pele/tissular prejudicada e Conforto prejudicado.

\section{Diagnósticos de risco e proposta de intervenções de enfermagem}

No local do presente estudo, ao término do procedimento cirúrgico, os pacientes permanecem em ventilação mecânica invasiva na SRPA. Essa condição favorece o "Risco de aspiração", e os fatores de risco evidenciados foram: tosse ineficaz, barreira à elevação superior do corpo, capacidade prejudicada para deglutir, cirurgia de cabeça/ pescoço, nível de consciência diminuído, reflexo de engasgo deprimido e também presença de sonda nasogástrica.

Dessa maneira, intervenções prioritárias são direcionadas, como a redução de fatores que possam levar à hipoxemia potencializada pelo efeito residual das drogas anestésicas utilizadas, principalmente pela condição clínica de pacientes oncológicos. Existem outros fatores que podem levar o paciente à diminuição da oxigenação tecidual, como: estado físico, idade acima de 55 anos, doença pulmonar obstrutiva crônica, diabetes mellitus, saturação de oxigênio no pré-operatório menor que $95 \%$, déficit nutricional, pacientes do sexo feminino e anestesia geral. ${ }^{(16-17)}$

O enfermeiro atuante na SRPA necessita ser capacitado para o planejamento da SAE e a execução das $\mathrm{AE}$ que favorecem a diminuição das complicações, prevenção de eventos adversos e minimização de riscos. ${ }^{(18)}$

O diagnóstico "Risco de infecção" foi relacionado aos fatores de risco como a alteração na integridade da pele, além da presença de acesso venoso periférico e central; incisão cirúrgica; 
drenos; sonda vesical de demora; via aérea artificial; cistostomia; nefrostomia e gastrostomia. Estudos que descreveram diagnósticos e intervenções de enfermagem para pacientes em POI de cirurgia eletiva, não relacionaram o diagnóstico de Risco de infecção. ${ }^{(18)}$ Porém, em uma revisão integrativa, que identificou artigos relacionados à assistência de enfermagem a pacientes críticos na SRPA, os autores consideraram que a incidência de infecção do sítio cirúrgico pode estar associada como complicações frequentes. ${ }^{(18)}$

Em relação ao diagnóstico "Risco de queda" foram identificados os seguintes fatores de risco: mobilidade prejudicada, utilização de drogas anestésicas no intraoperatório, permanência do paciente na SRPA, alteração na função cognitiva e nível de consciência diminuído. Para minimização desses fatores, foram selecionadas as IE de prevenção contra quedas e transferências, e, como atividades, determinar nível de consciência e a capacidade de cooperar, identificar comportamento e fatores que afetam o risco de queda, e planejar tipo e método de movimentação. ${ }^{(19)}$

\section{Diagnósticos com foco no problema e proposta de intervenções de enfermagem}

As características definidoras do diagnóstico "Capacidade de transferência prejudicada" foram: capacidade de transferir-se da cama para maca de transporte e capacidade de transferir-se da maca de transporte para a cama. Os fatores relacionados foram: a dor, o equilíbrio prejudicado, a força muscular insuficiente, a alteração na função cognitiva e prejuízo neuromuscular e musculoesquelético.

O paciente na SRPA possui um nível alto de dependência da equipe de enfermagem para a transferência entre leitos, sendo que esse procedimento deve ser feito em bloco com auxílio de, no mínimo, dois profissionais de enfermagem. Outros aspectos limitantes da movimentação no leito e entre leitos do paciente relacionam-se aos fatores de risco para queda, sinais verbais e não verbais de dor e alterações nos sinais vitais. Os dispositivos como sondas, cateteres, drenos e outros também contribuem para a dependência da enfermagem e da capacidade do paciente em mobilizar-se.

O diagnóstico de "Mobilidade no leito prejudicada" possui como característica definidora a capacidade prejudicada de se posicionar na cama. Os fatores relacionados identificados foram: dor; força muscular insuficiente, associada a agentes farmacêuticos; e prejuízo neuromuscular e musculoesquelético. Na prática diária, esse diagnóstico está relacionado com a restrição no leito e restrição na movimentação corporal do paciente devido ao procedimento cirúrgico, e à presença de dispositivos médicos.

Em estudo(4) ${ }^{(4)}$ sobre a mobilidade no leito prejudicada, esta também foi discutida como um dos DE mais frequentes. Os fatores relacionados são evidenciados pela condição operatória e pelo uso de sedativos, e apresentam-se como IE, a relevância da orientação e monitoração da mecânica corporal para prevenção de lesões em decorrência da limitação de movimento do paciente.

O diagnóstico "Integridade da pele/tissular prejudicada" apresentou as seguintes características definidoras: alteração na integridade da pele; punção venosa; incisão cirúrgica; drenos e cateteres em cavidade. Como fatores relacionados foram identificadas: hidratação, ferida operatória, secreções e excreções.

Um estudo sobre pós-operatório de cirurgias eletivas em hospital universitário identificou o diagnóstico de integridade da pele prejudicada, pelo rompimento da superfície da pele e invasão das estruturas do corpo, relacionada a fatores mecânicos. As intervenções propostas visaram à prevenção de novas lesões em um tecido já prejudicado, além de promover cuidados para reparação do mesmo. ${ }^{(20)}$ A Associação Brasileira de Enfermeiros de Centro Cirúrgico, Recuperação Anestésica e Centro de Material e Esterilização (SOBECC) propõe como intervenção a supervisão da pele e avaliação de curativo. Como resultados esperados cita a termorregulação e cicatrização de feridas como fatores importantes de avaliação. ${ }^{(1)}$

Quanto ao diagnóstico "Conforto prejudicado" as características definidoras foram: sensação 
de desconforto, desconforto com a situação, descontentamento com a situação, lamento e inquietação. Os fatores relacionados identificados foram o controle situacional insuficiente e as condições associadas ao regime de tratamento e que estão relacionadas ao acesso venoso periférico e central, incisão cirúrgica, drenos (sucção, silicone e de tórax), sonda vesical de demora, sonda nasogástrica, presença de via aérea artificial, cistostomia, nefrostomia e gastrostomia. ${ }^{(21)}$

As intervenções de enfermagem compreendem o controle do ambiente e da dor do paciente, assim como a redução da ansiedade. Como resultados de enfermagem evidenciamos a obtenção de um estado de conforto ambiental, físico e psíquicoespiritual. Os indicadores de resultados identificados foram: temperatura do ambiente, posição confortável, dificuldade respiratória, ansiedade, medo, dor relata e não relatada (suspiros, choros, inquietação) e humor deprimido.

Documentar o cuidado de enfermagem mediante um sistema de linguagem padronizada é assegurar uma comunicação uniforme, uma descrição da singularidade da enfermagem e o armazenamento de dados para fins educacionais na tomada de decisão e/ou para dados estatísticos. ${ }^{(22)}$

\section{Conclusão}

O mapeamento cruzado realizado a partir da análise das 187 fichas e das classificações da NANDA-I originou uma listagem com cinco diagnósticos de enfermagem de risco e 11 diagnósticos de enfermagem com foco no problema. Obteve-se $100 \%$ de frequência dos diagnósticos: Risco de aspiração; Risco de infecção; Risco de queda; Capacidade de transferência prejudicada; Mobilidade no leito prejudicada; Integridade da pele/tissular prejudicada e Conforto prejudicado. Para esses oito diagnósticos foram selecionados: 22 intervenções; 58 atividades; 23 resultados esperados e 48 indicadores de enfermagem.

Salienta-se a necessidade de estudos descritivos primários com enfoque nesse ambiente crítico e a utilização de linguagem padronizada de enfermagem, que contribuem para a construção do conhecimento especializado e desenvolvimento de protocolos assistenciais.

O presente estudo colabora para a autonomia e visibilidade da atuação do enfermeiro em SRPA e no contexto perioperatório, assim como a utilização da padronização dos DE, RE e IE no ensino da graduação em enfermagem. Para a pesquisa de enfermagem, o estudo fortalece e estimula a exploração do conhecimento e a utilização da linguagem padronizada.

\section{Agradecimentos}

As autoras agradecem o apoio do Conselho Federal de Enfermagem (Cofen) e da Coordenação de Aperfeiçoamento de Pessoal de Nível Superior (CAPES), por meio do Acordo Capes/Cofen Edital $n^{\circ}$ 27/2016 - Apoio a Programas de Pós-graduação da Área de Enfermagem - Modalidade Mestrado Profissional, o qual contemplou com recursos financeiros o projeto.

\section{Conflitos de interesse}

As autoras declaram não haver conflitos de interesse.

\section{Referências}

1 Sociedade Brasileira de Enfermeiros de Centro Cirúrgico, Recuperação Anestésica e Centro de Material e Esterilização. Diretrizes de práticas em enfermagem cirúrgica e processamento de produtos para a saúde. 7a ed. São Paulo: SOBECC; 2017.

2 Conselho Federal de Enfermagem. Resolução Cofen-358/2009. [Internet]. Brasília: COFEN; 2009. [citado 2021 Ago 18]. Disponível em: http://www.cofen.gov.br/resoluo-cofen-3582 009_4384.html

3 Pereira GN, Abreu RNDC, Bonfim IM, Rodrigues AMU, Monteiro LB, Sobrinho JM. Relationship between systematization of nursing care and patient safety. Enferm Foco. 
2017;8(2):21-5. Disponível em: http://revista. cofen.gov.br/index.php/enfermagem/article/ view/985

4 Silva HVC, Souza VP, Silva PCV. Systematization of perioperative nursing care in a postanesthetic recovery unit. Rev Enferm UFPE. 2016;10(10):3760-7. doi: 10.5205/reuol.966787805-1-ED1010201606.

5 Amaral JAB, Spiri WC, Bocchi SCM. Quality indicators in nursing with emphasis in the surgical center: integrative literature review. Rev SOBECC. 2017;22(1):42-51. doi: 10.5327/ Z1414-4425201700010008.

6 Horta WA. Processo de enfermagem. São Paulo: EPU-EDUSP; 1979.

7 Horta WA. Processo de enfermagem. Rio de Janeiro: Guanabara Koogan; 2011.

8 Herdman TH, Kamitsuru S. Diagnósticos de enfermagem da NANDA I: definições e classificação (2018-2020). 11a ed. Garcez RM, tradutora. Porto Alegre: Artmed; 2018.

9 Bulecheck GM, Butcher HK, Dochterman JM. Classificação das intervenções de enfermagem (NIC). 6a ed. Rio de Janeiro: Elsevier; 2016.

10 Moorhead S, Johnson M, Maas M. Classificação dos resultados de enfermagem: NOC. 5a ed. Rio de Janeiro: Elsevier; 2016.

11 Silva DVA, Sousa INM, Rodrigues CAO, Pereira FAF, Gusmão ROM, Araújo DD. Nursing diagnoses in a home-based program: cross-mapping and NANDA-I Taxonomy. Rev Bras Enferm. 2019;72(3):584-91. doi: 10.1590/0034-7167-2018-0323.

12 Cruz LF, Felix MMS, Ferreira MBG, Pires PS, Barichello E, Barbosa MH. Influence of socio-demographic, clinical and surgical variables on the Aldrete-Kroulik Scoring System. Rev Bras Enferm. 2018;71(6):3189-95. doi: 10.1590/0034-7167-2017-0813.

13 Riegel F, Oliveira Junior NJ. Nursing process: implications for the safety of surgical patients. Cogitare Enferm. 2017;22(4):1-5. doi: 10.5380/ ce.v22i1.45577.
14 Tannure MC, Salgado PO, Chianca TCM. Cross-Mapping: diagnostic labels formulated according to the ICNP ${ }^{\circledR}$ versus diagnosis of NANDA International. Rev Bras Enferm. 2014; 67(6):972-8. doi: 10.1590/0034-7167.201467 0616 .

15 Morais SCRV, Nóbrega MML, Carvalho EC. Cross-mapping of results and Nursing Interventions: contribution to the practice. Rev Bras Enferm. 2018;71(4):1993-2000. doi: 10.1590/ 0034-7167-2017-0324.

16 Saraiva EL, Sousa CS. Critically ill patients in the postanesthesia care unit: integrative review. Rev SOBECC. 2015;20(2):104-12. doi: 10.5327/Z1414-4425201500020006.

17. Mendonça FT, Lucena MC, Quirino RS, Govêia CS, Guimarães GMN. Risk factors for postoperative hypothermia in the post-anesthetic care unit: a prospective prognostic pilot study. Rev Bras Anestesiol. 2019;69(2):122-30. doi: 10.1016/j.bjane.2018.11.002.

18 Campos MPA, Dantas DV, Silva LSL, Santana JFNB, Oliveira DC, Fontes LL. Complications in the post-anesthesia care unit: an integrative review. Rev SOBECC. 2018;23(3):160-8. doi: 10.5327/Z1414-4425201800030008.

19 Bonetti AEB, Girardello DTF, Coneglian ALA, Egevardt D, Batista J, Cruz EDA. Assistance of the nursing team to the patient in postanesthetic recovery room. Rev Enferm UFSM. 2017;7(2):193-205. doi: 10.5902/2179 769226840 .

20 Bertoncello KCG, Sávio B, Ferreira JM, Amante LN, Nascimento ERP. Nursing diagnoses and proposals for nursing interventions for patients in the immediate post-operative period following elective surgery. Cogitare Enferm. 2014;19(3):582-9. Available from: https://revistas.ufpr.br/cogitare/article/ view/33676/23252

21 Gonçalves MCS, Brandão MAG, Duran ECM. Validation of the defining characteristics of the nursing diagnosis impaired comfort in oncology. Acta Paul Enferm. 2016;29(1):115-24. doi: 10.1590/1982-0194201600016. 
22 Ferreira AM, Rocha EN, Lopes CT, Bachion MM, Lopes JL, Barros ALBL. Nursing diagnoses in intensive care: cross-mapping and NANDA-I taxonomy. Rev Bras Enferm. 2016;69(2):307-15. doi: 10.1590/0034-7167. 2016690214 i.

Recebido em: 15 mar. 2021

Aceito em: 19 ago. 2021 
Prearo, M.; Vocci, M. C.; Fontes, C. M. B. 